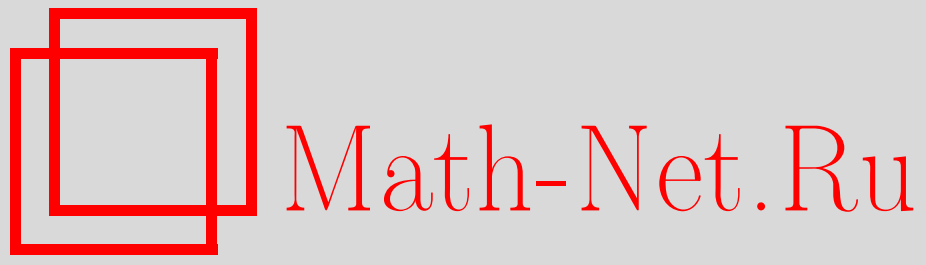

А. М. Вашевник, K определению обобщенных многочленов Чебышёва над конечными полями, Функи. анализ и его прил., 2001, том 35, выпуск 3, 77-79

DOI: https://doi.org/10.4213/faa262

Использование Общероссийского математического портала MathNet.Ru подразумевает, что вы прочитали и согласны с пользовательским соглашением

http://www . mathnet.ru/rus/agreement

Параметры загрузки:

IP : 107.22 .136 .117

26 апреля 2023 г., 14:47:09

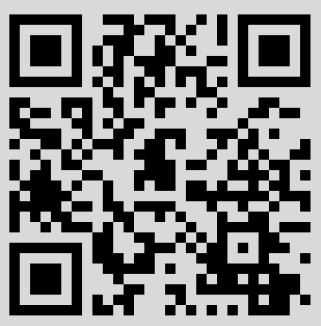


Теорему 1 можно рассматривать как обобщение на случай факторов известной теоремы из линейной алгебры о стандартном виде вещественной кососимметрической матрицы.

$$
\begin{aligned}
& \text { Пусть } s=\left(\begin{array}{cc}
s_{1} & 0 \\
0 & s_{2}
\end{array}\right) \text {. Тогда } \\
& k=\left(\begin{array}{ccccc}
0 & 0 & s_{1} & 0 \\
0 & 0 & 0 & s_{2} \\
-s_{1} & 0 & 0 & 0 \\
0 & -s_{2} & 0 & 0
\end{array}\right)=\left[\left(\begin{array}{cccc}
0 & 1 & 0 & 0 \\
-1 & 0 & 0 & 0 \\
0 & 0 & 0 & 0 \\
0 & 0 & 0 & 0
\end{array}\right),\left(\begin{array}{cccc}
0 & 0 & 0 & -s_{2} \\
0 & 0 & s_{1} & 0 \\
0 & -s_{1} & 0 & 0 \\
s_{2} & 0 & 0 & 0
\end{array}\right)\right] .
\end{aligned}
$$

Отсюда следует

ТЕОРЕмА 2. Всякий кососимметрический элемент вещественного фактора является коммутатором двух кососимметрических әлементов из обертывающцей алгебры фон Неймана.

СледствиЕ. Всякий кососимметрический элемент вещественного фактора $R$ можно представить в виде $\left[k_{1}, k_{2}\right]+\left[s_{1}, s_{2}\right]$, где $k_{1}, k_{2} \in R_{k}, s_{1}, s_{2} \in R_{s}$.

\title{
ЛИТЕРАТУРА
}

1. Fack Th., de la Harp P. Ann. Inst. Fourier, Grenoble, 30, No. 3, 49-70 (1980). 2. Ayupov Sh. A., Rakhimov A. A., Usmanov Sh. M. Jordan, Real and Lie Structures in Operator Algebras. Kluwer Acad. Publ., Dordrecht, 1997. 3. Størmer E. Pacif. J. Math., 21, No. 2, 349-370 (1967). 4. Аюпов Ш. А. Классификация и представление упорядоченных йордановых алгебр. Ташкент, Изд-во «ФАН», 1986. 5. Садовский В. Ю. Узб. матем. журн., No. 1, 55-63 (1994). 6. Ayuрол Sh. A., Azamov N. A. Comm. Algebra, 24, No. 4, 1501-1520 (1996).

Институт математики Академии Наук республики Узбекистан

Поступило в редакцию 22 июня 2000 г.

\section{К определению обобщенных многочленов Чебышёва над конечными полями}

\author{
(C) 2001. А. М. ВАшевНИК
}

1. Введение. Теория так называемых детских рисунков Гротендика, ведущая начало от работы [3], в настоящее время развивается довольно интенсивно. Часть этой теории связывает комбинаторно-топологические объекты (графы на поверхностях, которые и называются детскими рисунками) с алгебраическими «функциями Белого», определенными над полем комплексных чисел; см. [4].

Однако функции Белого имеют смысл и над произвольным полем и допускают независимое изучение.

В настоящей работе делается шаг в направлении распространения частного случая теории (в котором функции Белого сводятся к обобщенным многочленам Чебышёва) на произвольные алгебраически замкнутые поля. 
Оказывается, что определения, очевидным образом эквивалентные над $\mathbb{C}$, приводят к разным классам многочленов над конечными полями. В дальнейшем будут введены соответствующие определения, установлены связи между ними и приведены примеры, показывающие их неравносильность.

2. Обобщенные многочлены Чебышёва над $\mathbb{C}$. В теории графов на поверхностях и кривых над числовыми полями рассматриваются так называемые обобщенные многочлены Чебышёва (ОМЧ). По определению комплексный многочлен $P$ является ОМЧ, если для некоторых $c^{+}$и $c^{-}$прообраз отрезка $\left[c^{+}, c^{-}\right]$является деревом (связным графом без циклов). Множество таких многочленов будем обозначать через ОМЧ(즌. Известно, что каждое из следующих 5 условий эквивалентно тому, что многочлен $P$ степени $n$ лежит в ОМЧ(ㅈ).

Условие 1. Множество критических значений многочлена $P$ содержится в множестве $\left\{c^{-}, c^{+}\right\}$.

Условие 2. Множество корней многочлена $\operatorname{Discr}(P-c)$ содержится в множестве $\left\{c^{-}, c^{+}\right\}$.

Условие 3. Уравнение $P=c$ имеет $n$ различных корней при $c \neq c^{+} u c \neq c^{-}$.

Условие 4. $\left(P-c^{-}\right)\left(P-c^{+}\right) \vdots d P / d x$.

Условие $5 . m^{+}+m^{-}=n+1$, где $m^{ \pm}-$число корней многочлена $P-c^{ \pm}$ (без учета кратностей).

3. ОМЧ над произвольным алгебраически замкнутым полем. Пусть $F-$ алгебраически замкнутое поле, а $P$ - многочлен над этим полем. Пусть $n=$ $\operatorname{deg} P$ и $p=\operatorname{char} F$. Будем предполагать, что $p$ не делит $n$ (тогда $\operatorname{deg}(d P / d x)=$ $n-1)$. Верны следующие соотношения между условиями $1-5$ :

Tеорема 1. $1 \Longleftrightarrow 2 \Longleftrightarrow 3 \Longleftarrow 4 \Longleftarrow 5$.

Доказательство того, что $1 \Longrightarrow 5$, в случае $F=\mathbb{C}$ основано на следующем утверждении:

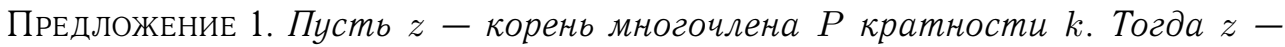
корень многочлена $d P / d x$ кратности $k-1$.

Оно верно лишь для $\operatorname{char} F=0$ (например, для $P=x^{n}+x^{p}$, где $p=\operatorname{char} F$, $n>p$, утверждение неверно). Однако в общем случае можно утверждать следующее:

Лемма 1. Пусть z-корень многочлена $P$ кратности $k$. Тогда если $k$ не делит $p$, то $z$ - корень многочлена $d P / d x$ кратности $k-1$, а если $k$ делит $p$, то $z$ - корень этого многочлена кратности не менее чем $k$.

ОСНовНОЕ ОПРЕДЕЛЕНИЕ. Многочлен $P$ называется ОМЧ в слабом смысле, если он удовлетворяет условию 1 , т. е. множество его критических значений содержится в множестве $\left\{c^{-}, c^{+}\right\} ; P$ называется ОМЧ в сильном смысле, если он удовлетворяет условию 5 , т. е. $m^{+}+m^{-}=n+1$. Пусть $P$ является ОМЧ в слабом смысле. Рассмотрим разложение $P-c^{ \pm}=\prod_{j=1}^{m^{ \pm}}\left(x-A_{j}^{ \pm}\right)^{v_{j}^{ \pm}}$, где $A_{j}^{ \pm} \in F$, $v_{j}^{ \pm} \in \mathbb{N}$ и все $A_{j}^{ \pm}$отличны друг от друга (такое представление существует и единственно, так как $F$ алгебраически замкнуто). Будем говорить, что обобщенному многочлену $P$ соответствует набор чисел $v_{j}^{ \pm}$, и введем обозначение $\operatorname{val}(P)=\left[v_{1}^{+}, v_{2}^{+}, \ldots, v_{m^{+}}^{+} \| v_{1}^{-}, v_{2}^{-}, \ldots, v_{m^{-}}^{-}\right]$. 
Теорема 2. Пусть Р является ОМЧ в слабом смысле. Тогда следующие условия эквивалентны:

(1) Р есть ОМЧ в сильном смысле;

(2) $v_{j}^{+} \neq 0(\bmod p), v_{j}^{-} \neq 0(\bmod p)$ для любого $j$.

\section{4. Примеры ОМЧ в слабом, но не в сильном смысле.}

1. $p=3, P=x^{4}+x, c^{-}=0, c^{+}=1$. В данном случае

$$
P=x(x+1)^{3}, \quad P-1=(x+1)(x-A)(x-B)(x-C),
$$

где все числа $A, B, C,-1$ различны, и $v^{+}=(1,3), v^{-}=(1,1,1,1)$. То есть многочлену $P$ соответствует набор чисел $[1,3 \| 1,1,1,1]$. Кроме того, $\operatorname{Discr}(P-c)=c^{3}$.

Теорема 3. Пусть $B \in \mathbb{Z}, 0<B<p, m \in \mathbb{Z}, m>0, u\left\{A_{i}\right\}_{i=1}^{m}-$ произвольный набор элементов из $F$, такой, что не все $A_{i}$ являются нулями. Тогда $P=x^{B} \sum_{i=1}^{m} A_{i} x^{p i}$ является ОМЧ в слабом смысле.

Для доказательства надо воспользоваться определением 2 и расписать дискриминант, учитывая, что $x(d P / d x)=B P$.

2. $P=x^{2 p+1}+x ; \operatorname{val}(P)=[p, p, 1 \| 1 \ldots 1]$.

3. $P=x^{2 p+1}+2 x^{p+1}+x ; \operatorname{val}(P)=[2 p, 1 \| 1 \ldots 1]$.

Tеорема 4. Пусть $B \in F, B \neq 0, n, m \in \mathbb{Z}, m>0, n>m p, u\left\{A_{i}\right\}_{i=1}^{m}-$ произвольный набор элементов из $F$. Тогда $P=B x^{n}+\sum_{i=1}^{m} A_{i} x^{p i}$ является ОМЧ в слабом смысле.

Доказательство проводится аналогично доказательству теоремы 3 с использованием равенства $d P / d x=B n x^{n-1}$.

Tеорема 5. Пусть $P(x)$ u $Q(x)$ являются ОМЧ в слабом смысле. Запишем разложения

$$
P(x)-c^{ \pm}=\prod_{j=1}^{m^{ \pm}}\left(x-A_{j}^{ \pm}\right)^{v_{j}^{ \pm}}, \quad Q(x)-c^{ \pm}=\prod_{j=1}^{s^{ \pm}}\left(x-C_{j}^{ \pm}\right)^{u_{j}^{ \pm}} .
$$

Пусть наборь $v_{j}^{+}$и $u_{j}^{+}$совпадают $u m^{+}=s^{+}=2$. Тогда совпадают наборь $v_{j}^{-} u u_{j}^{-}$.

Для доказательства нужно перевести $A_{j}^{+}$в $C_{j}^{+}$аффинным преобразованием; тогда $P$ перейдет в $Q$.

Автор благодарит Г. Б. Шабата за постоянное внимание и множество полезных советов при подготовке этой статьи.

\section{ЛИТЕРАТУРА}

1. Shabat G., Zvonkin A. In: Jerusalem combinatorics'93, Contemp. Math., Vol. 178, Amer. Math. Soc., Providence, RI, 1994, pp. 233-275. 2. Кострикин A. И. Введение в алгебру. Основы алгебры, Наука, M., 1994. 3. Grothendieck A. Esquisse d'un programme, London Math. Soc., Cambridge Univ. Press, 1997. 4. Shabat G. B., Voeyvodsky V. A. In: The Grothendieck Festschrift, Vol. III, Progr. Math., Vol. 88, Birkhauser, Boston, 1990, pp. 199227. 